\title{
Ziele \& Methodik der NextSkills Studie
}

Die NextSkills Studie verfolgt das Ziel, die unterschiedlichen Forschungsstränge zum Thema Future Skills aufzunehmen und empirisch zu untersuchen, wie diese für Hochschulen nutzbar gemacht werden können. Dafür wurde ein methodisch anspruchsvolles Design konzipiert. In diesem werden Skill Entwicklungen, Anforderungen und Bedarfe an zukünftige Kompetenzen aus der Praxis der Organisationen erhoben, indem umfangreiche qualitative Interviewdaten durch induktive Herangehensweise analysiert wurden. Die Ergebnisse, Hinweise auf Future Skills sowie auf die Anforderungen an eine zukünftige Hochschule, wurden dann durch Expertinnen und Experten aus Wissenschaft und Wirtschaft validiert. Das Verbinden von unterschiedlichen Methoden sowie unterschiedlichen Perspektiven verfolgt dabei das Ziel, Daten von größerer Reichweite und Präzision zu bekommen, die eine Formulierung der Skill Konstrukte in stärkerer inhaltlicher Tiefe und Gehalt ermöglichen.

\section{A 1.1 Forschungsziele: Einblick in die NextSkills}

Der Studie liegt die Erkenntnis zugrunde, dass Organisationen und die Arbeitswelt sich ändern. Zu einzelnen Bereichen liegen hierzu bereits Analysen vor, die in der NextSkills Studie erstmals unter dem Fokus zukünftiger Skill Anforderungen zusammengeführt wurde. Sie sind in Kapitel B 2 Theoretische Grundlagen für Future Skills oder die „Drift to Self-Organisation“, Kapitel B 4 Future Skills für Future Organisationen: Analyse zukünftiger Organisationsmodelle sowie Kapitel C 1 Zehn Sekunden, die über die Zukunft der Hochschulen entscheiden dokumentiert und bilden den Ausgangspunkt der NextSkills Studie. Die sich ständig wandelnde Ar- 
beits-, Lebens- und Lernkontexte bedingen, dass sich handelnde Akteurinnen und Akteure in Organisationen stetig weiterentwickeln und eine Anpassungsleistung vollbringen müssen, um weiterhin erfolgreich handeln zu können. Diese besteht darin, ihre Fähigkeit, erfolgreich mit ungewissen, komplexen Situationen umgehen zu können, beständig weiter zu entwickeln, also Kompetenzen aufzubauen (Erpenbeck et al. 2017). Eine Aktualisierung des Fachwissens reicht dabei nicht mehr aus. Aus der Analyse der vorhandenen Literatur liegen Hinweise vor, dass dem organisationalen Wandeln zu agilen, vernetzten, teamorientierten Organisationen, mit flachen Hierarchien und beteiligungsorientierten Führungsstrukturen eine explizite Vorstellung eines Mitarbeitertypus vorliegt, der u. a. dadurch gekennzeichnet ist, dass er Future Skills besitzt. Auf Basis der Literaturstudie wurde dann initial davon ausgegangen, dass diese u. a. durch folgende Charakteristika gekennzeichnet sind:

- ein hohes Maß an Selbstwirksamkeit,

- Fähigkeit, selbständig und autonom zu lernen,

- hoher Grad an Selbstorganisation in Bezug auf die eigene Arbeit,

- Reflexionsfähigkeiten zur eigenen Positionierung,

- Kommunikative Fähigkeit, eigene Ziele sowie eigene Bedürfnisse zu artikulieren.

Diese Charakteristika bildeten daher den Ausgangspunkt der zunächst qualitativen Untersuchung, in der weiterhin erfragt wurde, welche dieser und weiterer Aspekte für zukünftige Fähigkeitsanforderungen als

1. wichtig und relevant wahrgenommen werden,

2. durch explizite und beschreibbare Maßnahmen umgesetzt und unterstützt werden, und

3. wo Barrieren und Hindernisse existieren und wie damit umgegangen wird.

In der Untersuchung ging es darum, durch explorative, qualitative Vorgehensweisen ein Inventar insbesondere solcher Kompetenzen zu ermitteln, die Individuen bei der Bewältigung von Aufgaben und Gestaltung von Umgebungen in hoch agilen Arbeitsfeldern von Bedeutung sind. Zweitens, zu ermitteln welche Methoden und Verfahren aus Sicht der Organisationverantwortlichen sowohl als auch aus Sicht von Studierenden in Organisationen dazu geeignet sind, diese Fähigkeiten bei Mitarbeiterinnen und Mitarbeitern zu fördern ${ }^{3}$. Drittens darum, zu ermitteln, was Hochschulen dabei für eine Rolle spielen können.

3 In die Interviews wurden neben Personal- und Organisationsverantwortlichen auch dual Studierende in deren Praxisphase einbezogen. 
Abbildung 2 zeigt, dass sich die Untersuchung auf drei spezifische Fragestellungen konzentriert:

1. Welche Fähigkeiten sind dafür notwendig und wie können diese kompetenztheoretisch formuliert werden (Future Skills)?

2. Wie können Mitarbeiterinnen und Mitarbeiter dabei erfolgreich unterstützt werden?

3. Und wie kann die Hochschule diese Fähigkeiten bereits während des Hochschulstudiums unterstützen?

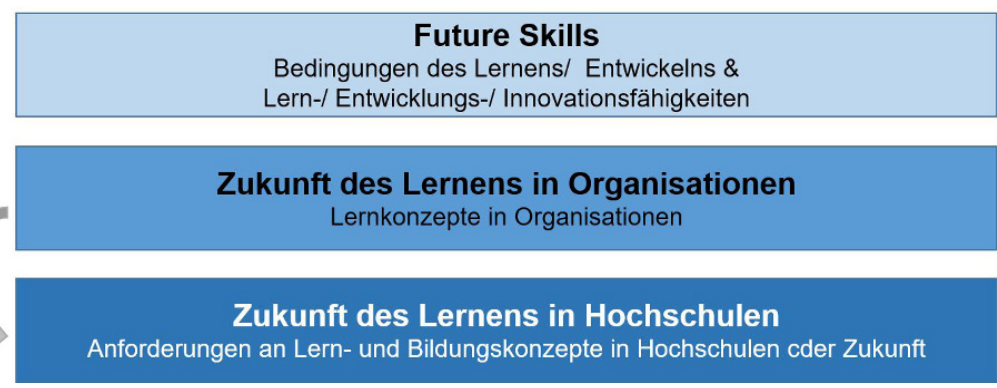

Abb. 2 Fragestellungen der NextSkills Studie

Die Untersuchung konzentriert sich in der empirischen Untersuchung auf sog. Future Organisations (siehe Kapitel A 1.3.1 Schritt 1: Identifikation von „Future Organisations"). Dies sind Organisationen, die bereits explizite, ausgearbeitete und entwickelte Konzepte der Personalentwicklung und ein explizites Verständnis der Förderung der Handlungskompetenz bei ihren Mitgliedern ausgedrückt haben. Um diese zu identifizieren, wurden Organisationen zunächst im Rahmen eines Wettbewerbs zum Thema Kompetenzorientierte Studienkonzepte durch kriteriengestützte Expertenevaluationen ausgewählt.

\section{A 1.2 Triangulation als methodologisches Leitkonzept}

Die Kombination von qualitativen und quantitativen Daten folgt dem Konzept der Triangulation, dass der Studie als methodologisches Leitkonzept zu Grunde liegt. Unter Triangulation versteht man die Kombination verschiedener Methoden, 
verschiedener Forschender, Untersuchungsgruppen, lokaler und zeitlicher Settings sowie unterschiedlicher theoretischer Perspektiven in der Untersuchung eines Phänomens (vgl. Denzin 1978). Die Voraussetzung einer solchen multidimensionalen Forschungsausrichtung ist es, quantitatives Material und qualitativ ermittelte Deutungen als prinzipiell gleichberechtigt anzusehen.

Denzin (ebenda) weist als einer der ersten darauf hin, dass eine Triangulation der Methoden bei der Untersuchung des gleichen Forschungsgegenstandes durch unterschiedliche Methoden die Validität durch Ermittlung kongruenter Ergebnisse betont. ${ }^{4}$ Dagegen hat die Kritik des Ansatzes von Denzin durch Wilson (1982) und später Lamnek (1988) den Validierungsaspekt in den Hintergrund treten lassen (vgl. dazu auch Sohr 1997). Seitdem werden die Ergebnisse triangulativ erhobener Daten eher als komplementär, das heißt als sich gegenseitig ergänzend, angesehen. Die ursprüngliche Intention Denzins (1978), mit der Triangulation verlässlichere und gültigeren Ergebnisse als bei Anwendung einer einzigen Forschungsmethode zu erzielen („Integrationsthese“, vgl. Treumann 1998 bzw. „Konvergenzmodell“, vgl. Kelle \& Erzberger 1999), ist also von der Einsicht abgelöst worden, dass die Methoden-Triangulation breitere und vielfältigere Erkenntnisse über die untersuchten Phänomene zu liefern imstande ist („Komplementaritätsthese“, vgl. Treumann 1998).

Sohr (1997) führt aus, dass Denzin (1978) vier Arten eines multimethodischen Vorgehens unterscheidet: die Datentriangulation (Nutzung unterschiedlicher Datenquellen für die Analyse), die Beobachtertriangulation (Einsatz unterschiedlicher Forschender zur Datenerhebung), die theoretische Triangulation (Anwendung unterschiedlicher Theorien auf den gleichen Gegenstand) und schließlich die Methodentriangulation. Diese kann als „within-method“ (zum Beispiel unterschiedliche Skalierungsverfahren innerhalb eines Methodensettings) und als „across-method “ (als Einsatz unterschiedlicher Methoden bei der Erfassung des gleichen Untersuchungsgegenstandes) angewendet werden. Ziel des Vorgehens ist es immer, „that the sociologist should examine his problem from as many methodological perspectives as possible.“ (ebenda: 297)

Erzberger (1995) vergleicht eine Untersuchung, die eine Verbindung von quantitativen und qualitativen Erhebungsverfahren herzustellen versucht, in anschaulicher Weise mit dem Bau einer Leiter,

„[...] wobei die beiden Holme die unterschiedlichen Methoden (standardisierte Erhebung und offenes Interviews) und die damit jeweils produzierten Ergebnisse darstellen, die durch Sprossen miteinander verbunden werden, und das heißt - soll

4 Ursprünglich stammt das Prinzip der Triangulation aus der Landvermessung, wo die Bestimmung der exakten Position eines Punktes in der Regel durch Messung aus mindestens zwei unterschiedlichen Positionen vorgenommen wird. 
die Leiter tragfähig sein - in beiden Holmen verankert werden müssen. Die Frage, wie die Sprossen genau aussehen, ob sie - übertragen auf den Forschungsprozess eigene Erhebungsschritte erforderlich machen und wo im Fortgang sie eingesetzt werden, ist nur aufgrund theoretischer Vorüberlegungen bzw. der zu untersuchenden Fragestellung zu beantworten. Die die qualitative und quantitative Erhebung gleichermaßen umfassende Forschungsfrage bildet somit - um im Bild zu bleiben - den Leim, der die einzelnen Teile der Leiter zusammenhält und diese erst begehbar macht." (ebenda: 43f.)

Das Forschungsdesign der NextSkills Studie (siehe Abbildung 3) ist gemäß dem methodologischen Leitprinzip der Triangulation als Kombination qualitativer und quantitativer Methoden konzipiert (siehe Abbildung 3 und Abbildung 4). Durch die Verknüpfung beider Methodenklassen soll gewährleistet werden, dass sich breitere, vielfältigere und tiefere Erkenntnisse über den untersuchten Gegenstandsbereich ergeben als bei der Anwendung nur einer einzigen Methode (gemäß der „Komplementaritätsthese“, vgl. Treumann 1998: 162). In der vorliegenden Arbeit ist das Konzept der Triangulation als Leitkonzept im Studiendesign verankert.

Im Sinne der Datentriangulation werden sowohl Daten und Ergebnisse aus Expertendiskussionen, qualitative Interviewdaten in Organisationen (Face-to-Face Interviews, unterschiedliche Zielgruppen: Experteninterviews, Interviews mit Lernenden), Daten aus Gesprächen mit Expertinnen und Experten zur Validierung und Daten aus der zweistufigen NextSkills Delphi-Studie miteinander trianguliert und zur Analyse eines Gegenstandes herangezogen: Der Entwicklung von Future Skills, der Definition von Lernmethoden, -prozessen und -konzepten und der Ermittlung von Ansatzpunkten zur Entwicklung von Hochschulen in der Zukunft. Im Sinne der Theorietriangulation wurden für die Betrachtung des Gegenstandes Future Skills Theorien der Bildungsforschung (zum strukturellen Bildungsbegriff, u. a. Meder 2006), der Kompetenzforschung (Definition und Operationalisierung von Kompetenz nach Erpenbeck et al. 2007), der Organisationsforschung (zur Emergenz Haken 1991 und zur Selbstorganisation Haken 2008) sowie der ökosystemische Ansatz Bronfenbrenners (1976 und 1981) herangezogen (zur Nutzung des ökosystemischen Modellansatzes in der empirischen Sozialforschung (siehe Epp 2018). Im Sinne der Methodentriangulation werden schließlich unterschiedliche qualitative Verfahren mit quantitativen Verfahren - sowohl bei der Datenerhebung als auch bei der Datenauswertung miteinander verknüpft („across method“). 


\section{Methodischer Aufbau}
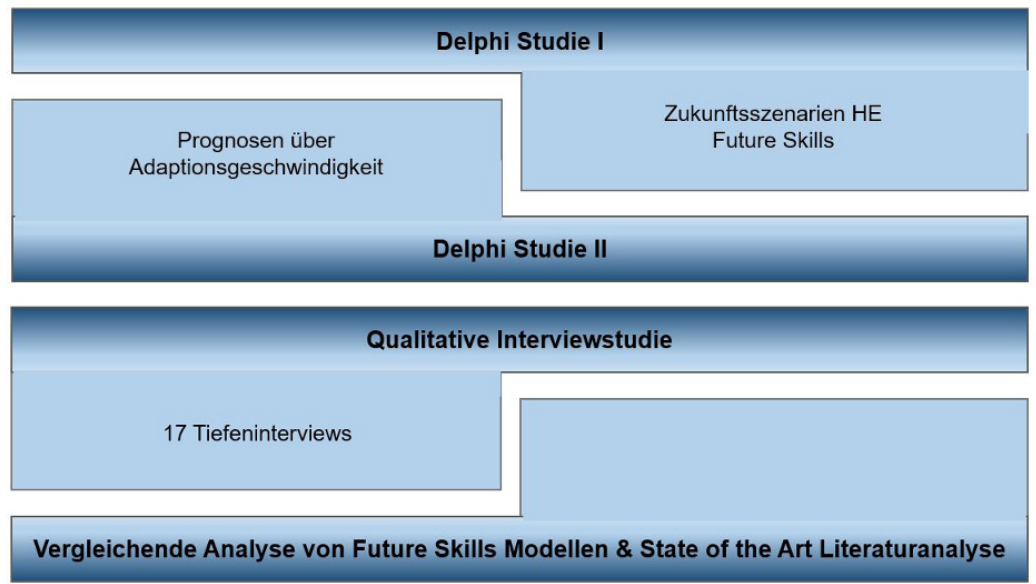

Expertengestütztes Screening und Analyse von Kompetenzkonzepten von 120 Organisationen

Abb. 3 Überblick über das Methodologische Design der NextSkills Studie

\section{A 1.3 Forschungsdesign}

Das Forschungsvorhaben NextSkills zielt darauf ab, zu analysieren, welche Fähigkeiten für eine produktive und proaktive Gestaltung zukünftiger Arbeitskontexte benötigt werden, um Anforderungen an Hochschulen abzuleiten (siehe Abbildung 3).

\section{A 1.3.1 Schritt 1: Identifikation von „Future Organisations”}

In einem ersten Schritt war es notwendig, solche Organisationen zu identifizieren, die bereits explizite Erfahrungen bei der Implementierung von Kompetenzmodellen, Vorstellung über Future Skills und einen hohen Reifegrad bei der Gestaltung zukünftiger Arbeitskontexte hatten. Dazu wurden sog. Future Organisations identifiziert, die als empirisches Feld geeignete Kontexte zur Ermittlung von Future Skills ausgeprägt haben. Das Auswahlverfahren fand im Jahr 2015 im Rahmen eines Wettbewerbs statt, bei dem über 8500 Partnerorganisationen der Dualen Hochschule Baden-Württemberg angeschrieben wurden und die Möglichkeit hatten, ihre Personalentwicklungs- und insbesondere ihre Konzeptionen für die Betreuung 
und Förderung von Studierenden einzureichen. An dem Wettbewerb ${ }^{5}$ beteiligten sich 124 Organisationen. Alle eingereichten Konzeptionen wurden im Rahmen eines kriteriengestützten Expertenratings bewertet. Die Kriterien zur Auswahl bezogen sich insbesondere auf die Analyse der Förderung von Handlungskompetenzen und internationalen Erfahrungen in den eingereichten Konzeptionen. Das so entstehende Ranking wurde dann im Rahmen einer Expertendiskussion von 15 Expertinnen und Experten diskursiv validiert und 20 Organisationen wurden im Rahmen einer Shortlist ausgewählt. Alle 20 Organisationen wurden eingeladen an der NextSkills Studie teilzunehmen, 17 reagierten positiv und wurden in das Interviewpanel eingeschlossen. Die Interviews fanden zwischen Dezember 2016 und Juni 2017 statt.

\section{A 1.3.2 Schritt 2: Interviewstudie}

Für die Interviewstudie wurden Leitfragen entwickelt, die zur Orientierung im Rahmen eines offenen, wenig strukturierten, problemvertiefenden Interviews eingesetzt wurden und sich auf folgende Aspekte fokussierte:

- Frage 1: Bitte erzählen mir Sie, in welcher Weise sich Lernen zukünftig in Organisationen vollzieht und welche Rolle dabei das selbstgesteuerte und selbstorganisierte, autonom initiierte und verantwortete Lernen, sowie Selbstwirksamkeit spielen?

- Frage 2: Lassen Sie uns einmal über Lernen nachdenken, wie es ihrer Erfahrung nach (wirklich) stattfindet.

- Frage 3: Erzählen Sie mir bitte, mit welchen Maßnahmen Sie Mitarbeiterinnen und Mitarbeiter dabei unterstützen?

- Frage 4: Welche Maßnahmen, Methoden und Lernmodelle eigenen sich dazu?

- Frage 5: Bitte erzählen Sie einmal, was Sie von einer Hochschule als Partner in der (vorbereitenden) Entwicklung und Unterstützung der Fähigkeiten von Mitarbeiterinnen und Mitarbeiter erwarten?

5 Der Wettbewerb war in Kooperation der Dualen Hochschule Baden-Württemberg, dem Ministerium für Wissenschaft Kunst und Kultur Baden-Württemberg und dem Arbeitsgeberverband Südwestmetall organisiert und als „Dualer Partner Award“ ausgeschrieben. 
Teilnehmende der Interviews waren die Personalverantwortlichen der Organisationen, und teilweise auch die Studierenden, die im Rahmen von dualen Studiengängen dort studierten. Insgesamt wurden 17 vertiefende Interviews geführt, an denen sich 20 Personen beteiligten und die zu etwa 700 Minuten qualitativem Interviewmaterial führten. Die Interviews wurden wortgetreu transkribiert und unter Anwendung der induktiven Kodierungstechnik (Mayring 1996; Thomas 2006) mit Hilfe der Software MaxQDA (VERBI Software 2017) von zwei Forschenden unabhängig kodiert. Passagen, die nicht einheitlich kodiert worden waren, wurden in einem zweiten Schritt diskutiert, um eine verlässliche Interrater-Reliabilität herstellen zu können. Ziel dabei war es, solche Konstrukte aus den Interviewdaten herauszuarbeiten, mit denen Bedingungen, Kontexte, Werte sowie Abläufe und Abhängigkeiten für zukünftig als wichtig erachteten Fähigkeiten bei Individuen rekonstruiert werden konnten. Zusätzlich wurden Konstrukte analysiert, die Aufschluss über die sich verändernden Arbeits- und Lernbedingungen in heutigen und zukünftigen Arbeits- und Lernkontexten rekonstruieren ließen. Auf diese Weise war es möglich, Dimensionen zukünftig wichtiger Fähigkeiten zu ermitteln, sowie deren Binnenbeziehung festzustellen und auf Basis inhaltlicher Nähe Fähigkeitsbündel zu sog. Future Skill Profilen zusammen zu stellen. In derselben Weise erlaubte der Analyseprozess die Rekonstruktion von durch die Befragten prognostizierten Bedingungen zukünftigen Wandels in Organisationsabläufen, sowie die Verortung von organisationalen Reaktionen, um dadurch entstehende Spannungen auszugleichen. Schließlich konnten Erwartungen und ausgedrückte Anforderungen an akademische Qualifizierungssysteme, wie Hochschulpartnerschaften, u. a. auch im dualen Studium, erhoben und zusammengestellt werden. Dies verschaffte Einblicke in die unterschiedlichen Dimensionen des durch digitale und vernetzte globale Kollaborationsprozesse ausgelösten Wandels innerhalb von Organisationen und skizzierte eine Reihe potentieller Szenarien für die künftige Hochschulbildung. Über ein kleines Sample von insgesamt 3 weiteren Interviews wurden die gewonnenen Konstrukte und die Hauptaussagen sowie die ermittelten Future Skills noch einmal qualitativ validiert. 


\section{A 1.3.3 Schritt 3: Internationale Delphi-Studie}

Um die qualitativ erworbenen Ergebnisse weiter zu verfeinern, sowie zu validieren wurde eine Delphi-Studie mit einem internationalen Expertenpanel durchgeführt. Die Delphi-Studie (zur Delphi Methodologie s. Dalkey \& Helmer 1963) mit dem Titel „Future Skills - Future Learning and Future Higher Education“ (Ehlers \& Kellermann 2019) umfasste 2 Befragungsrunden (siehe Abbildung 4). Zur Studie wurden 53 internationale Expertinnen und Experten aus unterschiedlichen Organisationen und Institutionen zur Teilnahme eingeladen. Diese arbeiteten in Hochschulen, als Forschende im Bereich Pädagogik, in Netzwerken, die sich mit Themen rund um Lernen, Digitalisierung der Hochschullehre und Skills Entwicklung befassen oder in Nichtregierungsorganisationen (NGO) (ebenda). Bei der Auswahl der Expertinnen und Experten wurde besonderes Augenmerk daraufgelegt, beide Perspektiven die der Hochschulen und die der Praxis - zu berücksichtigen. Außerdem wurde darauf geachtet, innerhalb dieser beiden Teilstichproben - Wissenschaft und Praxis - Expertinnen und Experten zu inkludieren, die verschiedene Positionen innerhalb ihrer Organisationen besetzen. Hierdurch sollte sichergestellt werden, dass ein Maximum an Differenzierung und Pluralität hinsichtlich unterschiedlicher Meinungen bezüglich der Themen - Zukunft des Lernens, der Skills und Hochschulbildung - vorherrschte, um die vollständige Breite des Erfahrungs- und Meinungsspektrums abzubilden und blind spots soweit wie möglich zu vermeiden. Insgesamt nahmen 49 Expertinnen und Experten an der ersten Runde und 40 an der zweiten Runde teil, die aus insgesamt siebzehn Ländern stammten (Australien, Österreich, Belgien, Kanada, Frankreich, Deutschland, China, Italien, Litauen, den Niederlanden, Neuseeland, Norwegen, Portugal, Spanien, Schweden, der Schweiz und dem Vereinigten Königreich).

Die Delphi-Studie wurde in zwei konsekutiv aufeinanderfolgenden Runden durchgeführt, wobei die zweite Erhebungsrunde mit einem zeitlichen Abstand von vier Wochen nach der ersten Runde erfolgte. In der ersten Runde lag der Fokus darauf, auf Basis der Einschätzungen des Samples im Konsens Konzepte, Definitionen und die Terminologie zu schärfen sowie deren Wichtigkeit zu klären. In der zweiten Runde sollten Expertinnen und Experten dann Einschätzungen abgeben, wie schnell die in der ersten Runde spezifizierten Komponenten im Hochschulkontext relevant werden würden. 


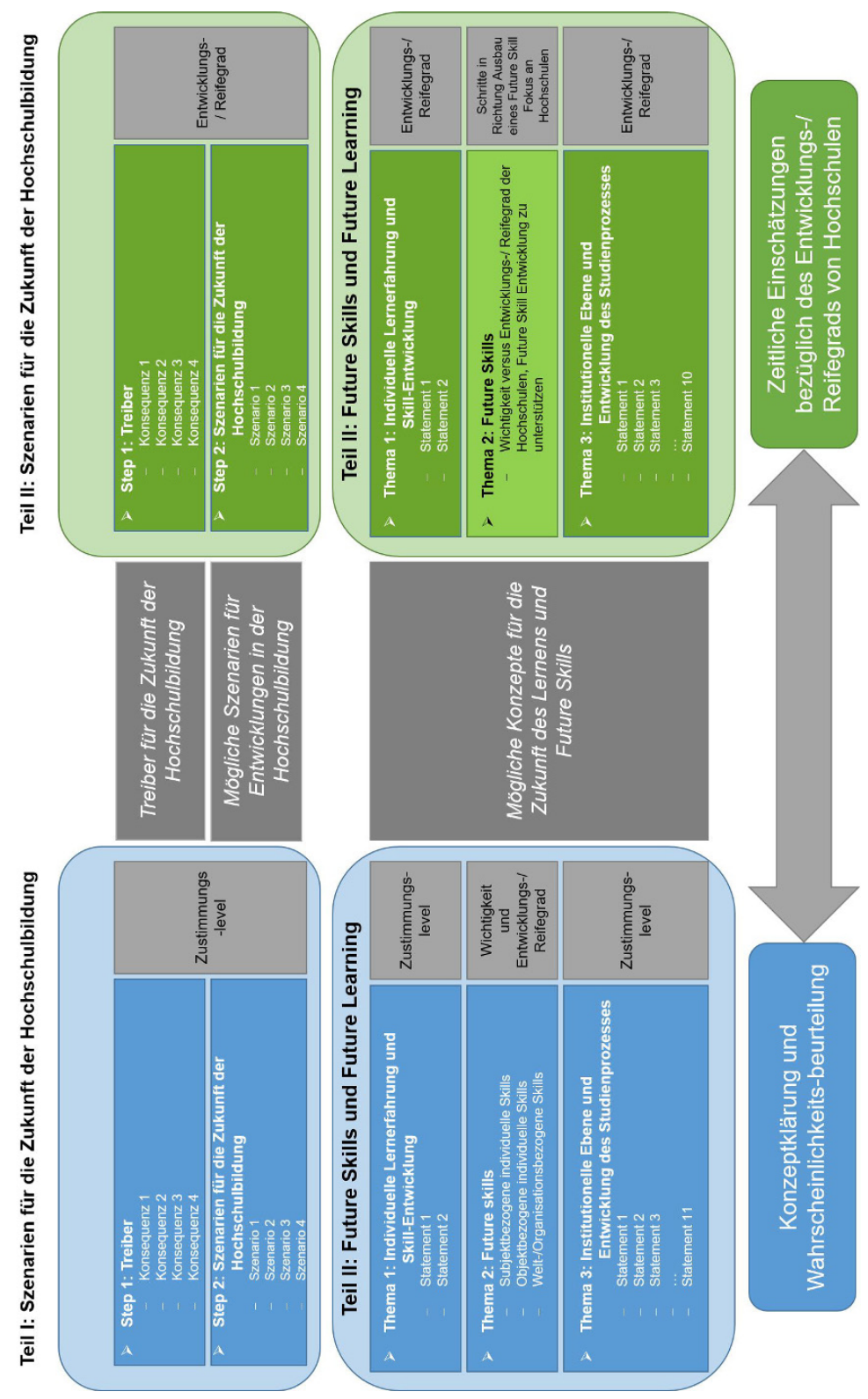

Abb. 4 Design der Delphi-Studie (Quelle: Ehlers \& Kellermann 2019) 
Abbildung 4 zeigt die Struktur und Logik des Fragebogens, die verschiedenen thematischen Bestandteile der einzelnen Befragungswellen und wie diese aufeinander aufbauen. Zentral war in beiden Runden, die Sichtweisen der Teilnehmenden zu Fähigkeiten, Prozessen, Strategien, Skills und Kompetenzen zu erfragen, welche künftige Arbeitnehmerinnen und Arbeitnehmer benötigen, um produktiv mit den sich ständig und immer schneller wandelnden organisationalen Realitäten der Zukunft umgehen zu können. Die qualitativen Kommentare des Samples sowie die Einschätzungen der Relevanz wurden analysiert und gingen in Form von verbesserten und reformulierten, geschärften Statements in die zweite Befragungswelle des Delphis ein (Runde 2). 